\title{
Estudio morfométrico de Quercus sartorii y Q. xalapensis (Fagaceae)
}

\author{
Morphometric study of Quercus sartorii and Q.xalapensis (Fagaceae) \\ Dorismilda Martínez-Cabrera ${ }^{1}$, Fernando Zavala-Chávez ${ }^{2 \dagger}$ y Teresa Terrazas ${ }^{3 *}$ \\ ${ }^{1}$ Programa de Botánica, Colegio de Postgraduados. Montecillo 56230 Estado de México, México. \\ ${ }^{2}$ División de Ciencias Forestales, Universidad Autónoma Chapingo. Chapingo 56230 Estado de México, México. \\ ${ }^{3}$ Instituto de Biología, Universidad Nacional Autónoma de México. Apartado postal 70-233, 04510 México D.F., México. \\ *Correspondencia: tterrazas@ibiologia.unam.mx \\ ${ }^{\dagger}$ Fallecido durante el periodo de revisión del artículo
}

\begin{abstract}
Resumen. Se realizó un estudio morfométrico con la finalidad de contribuir en la delimitación taxonómica de Quercus sartorii y Q.xalapensis. Para ello, se revisó material de herbario y se recolectó material en 11 poblaciones distribuidas en los estados de Hidalgo, San Luis Potosí, Tamaulipas y Veracruz, en México, incluyendo 2 poblaciones simpátricas. Los resultados del análisis de componentes principales y discriminante mostraron que los caracteres que contribuyeron a agrupar los individuos en su respectiva especie son los relacionados con el tamaño del fruto, en particular el diámetro de la bellota, de la nuez y de la cúpula. La bellota de $Q$. sartorii es de menor tamaño que la de Q. xalapensis. La variación detectada no se atribuyó a la latitud o altitud. El fenograma mostró la separación de 2 grupos que corresponden a los individuos de las 2 especies estudiadas, si los caracteres del fruto son incluidos. Además, de los caracteres cuantitativos ya mencionados, 3 del patrón de venación y 2 anatómicos contribuyeron a reconocer los individuos de cada especie. Estas diferencias se mantuvieron en los individuos de las poblaciones simpátricas, por lo que los resultados apoyaron la distinción de Quercus sartorii y Q. xalapensis.
\end{abstract}

Palabras clave: análisis multivariado, bellotas, México, patrón de venación, variación morfológica.

\begin{abstract}
A morphometric study was carried out in order to evaluate morphological variation of Quercus sartorii and Q. xalapensis and to contribute to their taxonomic delimitation. Herbarium specimens and material of 11 populations from Mexico in the states of Hidalgo, San Luis Potosí, Tamaulipas, and Veracruz, including 2 sympatric populations, were studied. Characters from foliar architecture, fruits and anatomical features of periderm and wood were analyzed through multivariate and phenetic methods. Principal component and discriminant analyses showed that fruit characters such as acorn, nut, and cupule diameters contributed to group individuals in their species, the acorn of $Q$. sartorii being smaller than that of $Q$.xalapensis. Variation was not attributed to latitude or elevation. The resulted phenogram revealed 2 groups in which individuals grouped in their own species if fruit features were included. In addition to the quantitative characters mentioned, 3 traits of the venation pattern plus 2 anatomical features distinguished the individuals of each species. These differences were maintained in the individuals from sympatric populations, supporting the distinctness of Quercus sartorii and Q. xalapensis.
\end{abstract}

Key words: acorn, Mexico, morphological variation, multivariate analyses, venation pattern.

\section{Introducción}

El género Quercus incluye árboles y arbustos en un número estimado de 400 (Nixon, 1997) a 500 especies (Manos et al., 1999) presentes en una amplia diversidad de hábitats en el hemisferio norte. En México cuenta con alrededor de 161 especies comprendidas en las secciones Lobatae, Protobalanus y Quercus, de las cuales 109 son endémicas de México (Valencia, 2004). Las especies mexi-

Recibido: 26 junio 2009; aceptado: 30 abril 2010 canas del género Quercus se encuentran distribuidas desde climas cálidos hasta templados, donde predominan junto con los pinos y constituyen la mayor parte de la cubierta vegetal de esos lugares (Rzedowski, 1978).

La sistemática de Quercus ha sido difícil de estudiar (Jensen et al., 1993) debido a la diversidad morfológica que presenta el género, asociada a la variabilidad dentro de las especies y la aparente facilidad de formar híbridos, lo cual puede generar morfologías intermedias que dificultan la identificación taxonómica entre especies (Palmer, 1948; Cristofolini y Crema, 2005; Albarrán-Lara et al., 2010; Peñaloza-Ramírez et al., 2010). Valencia (2004) menciona que la resolución de diversos problemas taxonómicos ha 
sido posible gracias al estudio particular de algunas especies mexicanas, entre las que podemos citar Q. acutifolia Née y $Q$. conspersa Benth.; $Q$. laceyi Small y $Q$. glaucoides M. Martens et Galeotti (Nixon y Muller, 1992); la nueva especie $Q$. hirtifolia M. L. Vázquez-Villagrán, S. Valencia A. et Nixon y su comparación con $Q$. hintoniorum Nixon et Muller, $Q$. hypoxantha Trel., $Q$. eduardii Trel., $Q$. laurina Humb. et Bonpl. y Q. depressa Humb. et Bonpl. (Vázquez et al., 2004); así como el complejo Q. hypoxantha Trel. (Nixon y Muller, 1993).

Sin duda estos estudios reflejan el problema del género en el nivel específico, como es el caso de las especies aquí estudiadas: $Q$. sartorii Liebm. y $Q$. xalapensis Humb. et Bonpl., clasificadas por Trelease (1924) en el subgénero Lobatae dentro de la serie Acutifoliae. Este autor reconoce Q. sartorii por la presencia de lenticelas de color café en las ramas, hojas elíptico-lanceoladas con ápice agudo, base típicamente redondeada, margen con dientes aristados y bellota de $\leq 1.5 \mathrm{~cm}$ de largo con cúpula en forma turbinada y más o menos esférica. En contraste, $Q$. xalapensis tiene lenticelas blancas en las ramas, hojas ovado-lanceoladas, margen aserrado, ápice agudo, base típicamente aguda y bellota de $>2 \mathrm{~cm}$ de largo con cúpula hemisférica no turbinada. Trelease (1924) también describió $Q$. xalapensis f. jalapae y $Q$. xalapensis f. surculina, haciendo referencia al largo y ancho de la hoja y al largo del pecíolo. Valencia (2004) propuso una lista preliminar de especies del género Quercus en México, donde reconoce $Q$. sartorii y $Q$. xalapensis dentro de la sección Lobatae, considerando $Q$. huitamalcana Trel. y $Q$. serra Liebm. como coespecíficas con $Q$. sartorii, y señala que las formas $Q$. xalapensis $\mathrm{f}$. jalapae y $Q$. xalapensis $\mathrm{f}$. surculina, propuestas por Trelease (1924), deben incluirse bajo Q. xalapensis. Martínez-Cabrera et al. (2003) registran caracteres de la arquitectura foliar y de la madera que apoyan el reconocimiento de ambas especies. Sin embargo, en la revisión taxonómica del complejo Acutifoliae, Romero (2006) considera que no existen diferencias claras que permitan distinguirlas, reconociendo a $Q$. sartorii coespecífica con Q. xalapensis. La variación morfológica de estos taxones ha causado confusión en su reconocimiento, por lo que se realizó un análisis morfométrico para evaluar y comparar la variación morfológica de ambas especies, así como determinar cuáles caracteres permiten delimitar cada taxón y si la altitud y la latitud de sus áreas de distribución influyen en dicha variación.

\section{Materiales y métodos}

Se recolectaron 47 individuos de $Q$. sartorii y $Q$. xalapensis provenientes de 11 poblaciones de los estados de Hidalgo, San Luis Potosí, Veracruz y Tamaulipas (Apéndice 1). En 2 localidades del estado de Tamaulipas se recolectaron poblaciones simpátricas para los taxones estudiados (Fig. 1), y se revisaron 119 ejemplares de herbario de ambos taxones, incluyendo el material tipo en la página electrónica de New York Botanical Garden (htpp://www. nybg.org/bsci/herbarium-imaging/ typedefinition.html) y en la del Missouri Botanical Garden (htpp://www. mobot.mobot.org./w3r/search/ image.fr/html), de los cuales únicamente 22 presentaron caracteres reproductivos. Estos ejemplares representan el área de distribución de las especies (Fig. 1).

De las muestras recolectadas en campo se seleccionaron 5 hojas y 5 frutos por individuo de cada población visitada, y del material de herbario, 3 hojas y los frutos disponibles de cada ejemplar. La forma en que se midieron algunos de los caracteres que resultaron importantes para separar los taxones se ilustra en la figura 2. Al final, se obtuvo información de 23 caracteres cuantitativos y 20 cualitativos (Apéndice 1). En adición a los caracteres vegetativos y reproductivos tradicionalmente empleados para la identificación de las especies de encinos, se incluyeron el patrón de venación y los caracteres anatómicos de la corteza y madera de ramas que describieron Martínez-Cabrera et al. (2003).

Análisis de datos. Los caracteres se analizaron por medio de métodos multivariados y de similitud. Para los análisis multivariados a todos los caracteres se aplicó la prueba de normalidad. Los caracteres que expresan distancia se transformaron con logaritmo natural; al número de estomas y de aréolas se les aplicó raíz cuadrada, y los grados se transformaron a radianes (Zar, 1999).

El análisis de componentes principales (ACP) incluyó 23 caracteres cuantitativos (Cuadro 1) y su objetivo fue sintetizar en un menor número de variables el mayor porcentaje de la variación (Neff y Marcus, 1980). El análisis discriminante (DISCRIM) se aplicó para estimar las tasas de error y porcentaje de individuos clasificados dentro de cada uno de los grupos (especies) reconocidos $a$ priori. Este análisis permitió corroborar si los individuos estudiados fueron asignados correctamente a los grupos propuestos (Johnson y Wichern, 1982).

Los análisis de varianza se realizaron con el modelo general lineal (GLM), adecuado para conjuntos de datos no balanceados. Estos análisis fueron seguidos de una prueba de comparación múltiple de medias (Tukey, 95\% de probabilidad) con la finalidad de detectar diferencias significativas intra e interespecíficas. Se aplicó un análisis de correlación con la finalidad de conocer el grado de asociación entre la altitud o la latitud con los caracteres morfológicos evaluados. Todos los análisis se practicaron con el paquete estadístico SAS (SAS Institute, 1989). 


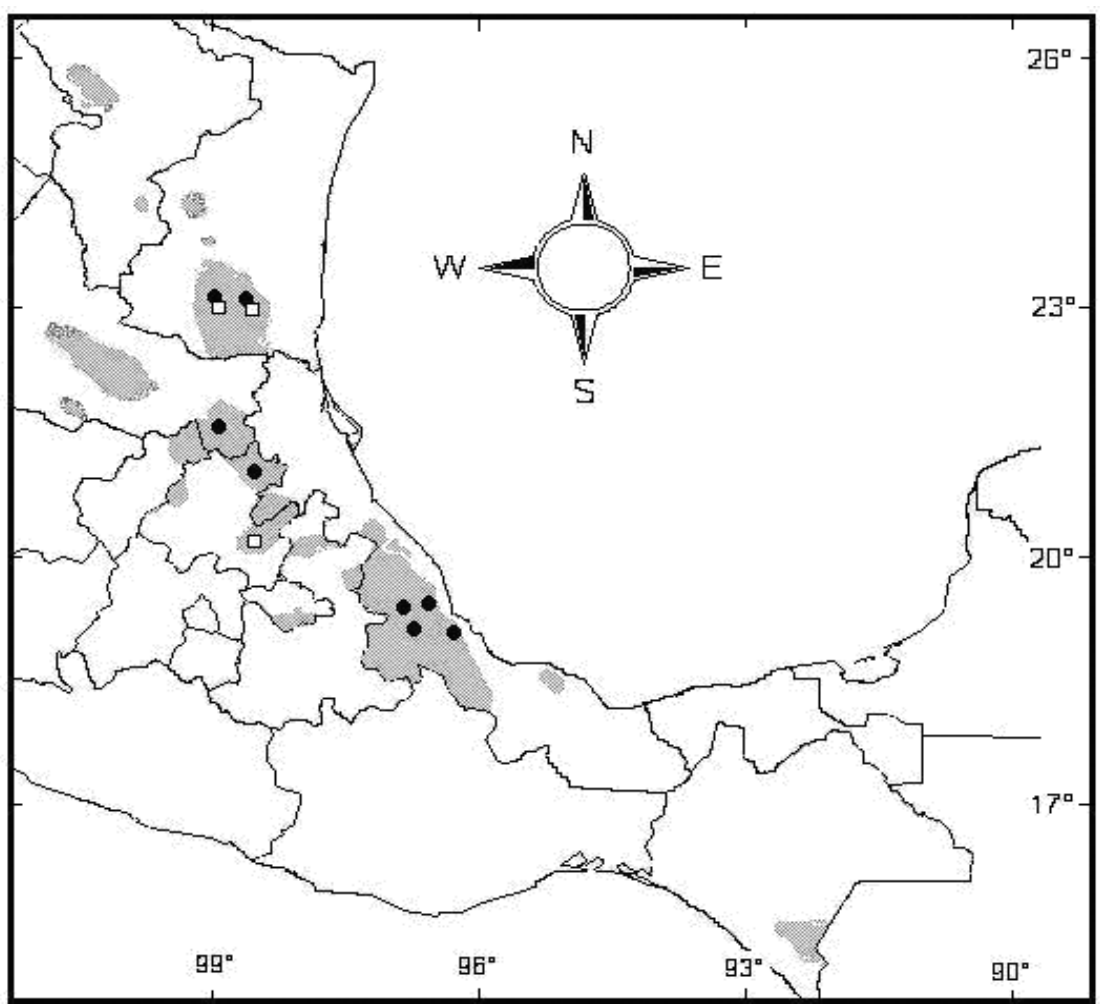

Figura 1. Distribución geográfica de las 11 poblaciones de Quercus sartorii $(\square)$ y $Q$. xalapensis $(\bullet)$ recolectadas. El sombreado representa el área de distribución para ambas especies con base en la revisión de ejemplares de herbario.
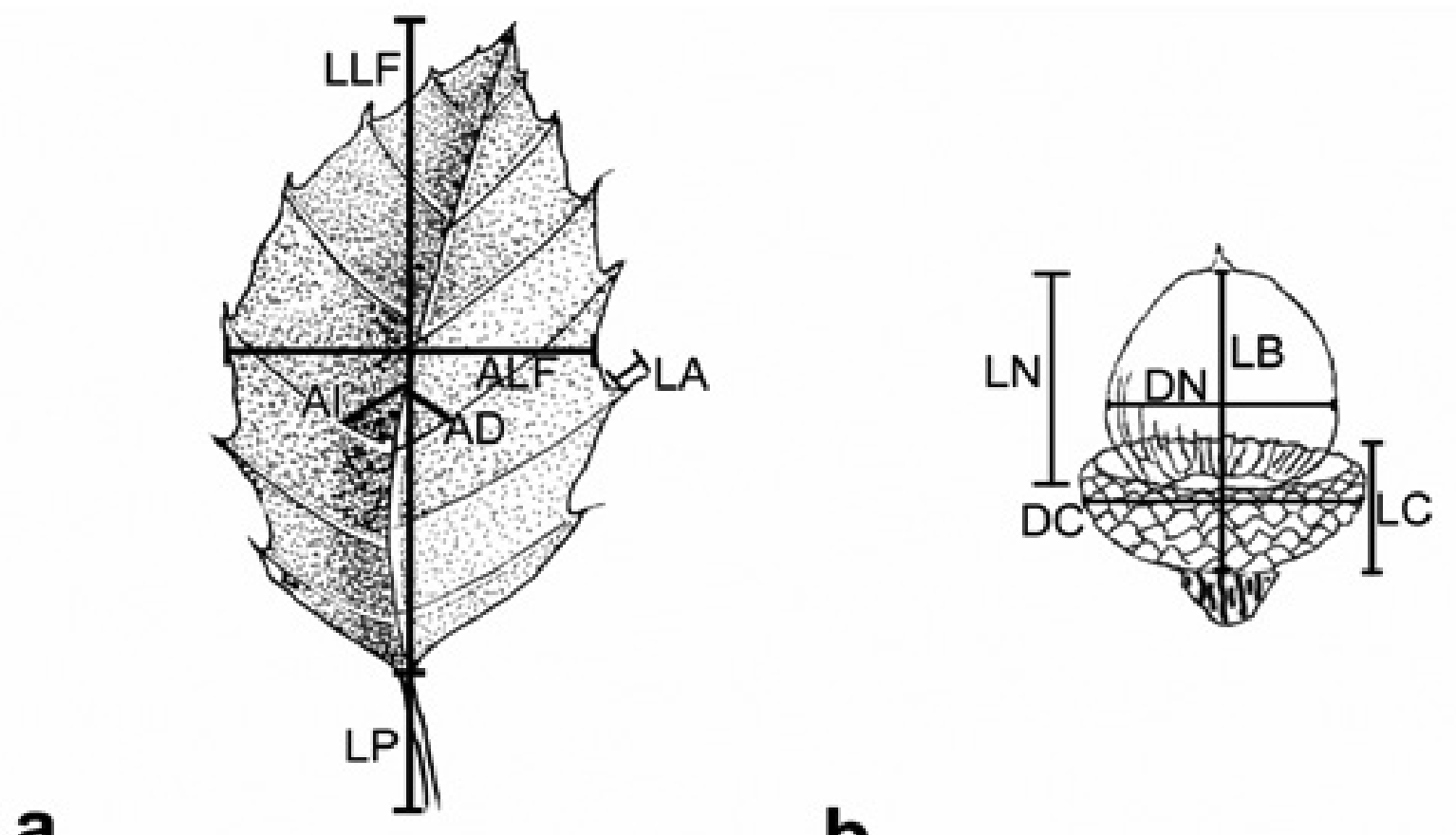

Figura 2. Diagrama que ilustra los caracteres medidos en la hoja y el fruto de Quercus sartorii y Q. xalapensis. a, lámina foliar; b, fruto. LLF, longitud de la lámina foliar; ALF, ancho de la lámina foliar; LP, longitud del pecíolo; AD, ángulo medio de la parte derecha; AI, ángulo medio de la parte izquierda; LA, longitud de aristas; LB, longitud bellota; LN, longitud nuez: LC, longitud cúpula; DN, diámetro de la nuez; DC, diámetro de la cúpula. 
Cuadro 1. Resultados del ACP para los 3 primeros componentes del material colectado para las especies de Quercus estudiadas considerando 23 caracteres

\begin{tabular}{|c|c|c|c|}
\hline \multirow[t]{2}{*}{ Caracteres } & \multicolumn{3}{|c|}{ Componentes } \\
\hline & Prin 1 & Prin2 & Prin3 \\
\hline Variación explicada (\%) & 29.56 & 15.27 & 9.20 \\
\hline Valor de carga & 6.20 & 3.20 & 1.93 \\
\hline Largo de hoja & 0.153 & $0.358 *$ & 0.318 \\
\hline Ancho de la hoja & 0.156 & 0.328 & 0.339 \\
\hline Lago del pecíolo & 0.024 & 0.021 & $0.489^{*}$ \\
\hline Diámetro del pecíolo & 0.070 & 0.255 & 0.085 \\
\hline Largo de las aristas del lado derecho de la lámina foliar & -0.128 & -0.133 & 0.409 \\
\hline Largo de las aristas del lado izquierdo de la lámina foliar & -0.071 & -0.084 & 0.401 \\
\hline Ángulo medio de la parte derecha de la lámina & 0.230 & 0.169 & 0.092 \\
\hline Ángulo medio de la parte izquierda de la lámina & 0.251 & 0.125 & 0.070 \\
\hline Largo de las yemas & -0.112 & 0.376 & -0.165 \\
\hline Ancho de las yemas & -0.069 & 0.290 & -0.175 \\
\hline Largo de la bellota & 0.253 & 0.095 & 0.137 \\
\hline Diámetro de la bellota & $0.368^{*}$ & -0.077 & -0.077 \\
\hline Largo de la cúpula & 0.240 & 0.039 & -0.209 \\
\hline Diámetro de la cúpula & $0.359^{*}$ & -0.056 & -0.016 \\
\hline Largo de la nuez & 0.314 & 0.114 & -0.075 \\
\hline Diámetro de la nuez & $0.328^{*}$ & 0.025 & -0.076 \\
\hline Diámetro de la cicatriz de la nuez & 0.325 & -0.016 & -0.168 \\
\hline Largo del pedúnculo & 0.009 & 0.090 & 0.023 \\
\hline Proporción del largo/diámetro de la bellota & -0.131 & 0.255 & 0.017 \\
\hline Número de aréolas & -0.171 & $0.423 *$ & -0.103 \\
\hline Número de estomas & -0.216 & 0.345 & -0.138 \\
\hline Longitud de las células oclusivas & -0.103 & 0.234 & -0.156 \\
\hline Número de células de la felodermis & 0.245 & 0.319 & -0.348 \\
\hline
\end{tabular}

* Variables con valores más altos. 
Se hicieron 2 análisis de similitud con el paquete NTSYSpc versión 2.0 (Rolhf, 1997). El primero consistió en analizar una matriz de datos con 43 líneas (caracteres, Apéndice 2) y 47 columnas (individuos), donde únicamente se incluyó el material recolectado. En el segundo análisis se utilizó una matriz de datos con 43 líneas (caracteres) y 164 columnas (individuos); aquí se incluyó el material recolectado y los ejemplares procedentes de los herbarios, que en su mayoría fueron estériles. Para reducir los efectos de las diferentes escalas en las mediciones, las matrices se estandarizaron por líneas, utilizando para ello la media y la desviación estándar del programa NTSYS. La matriz de similitud entre las unidades operacionales taxonómicas (OTU) se generó con el coeficiente de distancia euclideana y para la construcción de los fenogramas se empleó el método de agrupamiento promedio no ponderado (UPGMA) (Sneath, 1976).

\section{Resultados}

Los resultados del ACP mostraron que los 3 primeros componentes expresaron el $54.3 \%$ de la variación. Los caracteres que explicaron la variación del primer componente son los del fruto, diámetro de la bellota, cúpula y nuez. En el segundo y tercer componente están el largo de la hoja, número de aréolas y largo del pecíolo (Cuadro 1). El DISCRIM detectó que el $97.5 \%$ de los individuos de Quercus sartorii se clasificaron correctamente en esta especie y únicamente el $2.5 \%$ se agrupó con $Q$. xalapensis. Los 2 individuos clasificados incorrectamente correspondieron a la población de Alta Cima, Tamaulipas, donde ambas especies son simpátricas. Para Q. xalapensis el $99.2 \%$ de los individuos se clasificaron correctamente dentro de esta especie y solamente un individuo, también de la población de Alta Cima, Tamaulipas, se agrupó con los individuos de $Q$. sartorii.

Los resultados del análisis de correlación mostraron que algunos de los caracteres vegetativos de Quercus sartorii y $Q$. xalapensis estuvieron correlacionados con la latitud (L) y la altitud (A). En Q. sartorii, el largo de la hoja $[r(\mathrm{~L})=0.52, \mathrm{P}<0.0001 ; r(\mathrm{~A})=-0.58, \mathrm{P}<0.0001]$, el largo de las yemas $[r(\mathrm{~L})=0.59, \mathrm{P}<0.0001 ; r(\mathrm{~A})=-0.54$, $\mathrm{P}<0.0001]$ y el número de estomas $/ \mathrm{mm}^{2}[r(\mathrm{~L})=0.97$, $\mathrm{P}<0.0001 ; r(\mathrm{~A})=-0.90, \mathrm{P}<0.0001]$ estuvieron correlacionados positivamente con la latitud y negativamente con la altitud. Sin embargo, la longitud de las células oclusivas presentó una asociación negativa con la latitud $(r=-0.85, \mathrm{P}<0.0001)$ y positiva con la altitud $(r=0.79$, $\mathrm{P}<0.0001)$. En $Q$. xalapensis, el largo del pecíolo $[r(\mathrm{~L})=$ $-0.33, \mathrm{P}<0.0001 ; r(\mathrm{~A})=0.36, \mathrm{P}<0.0001]$, el ángulo del lado derecho de la lámina foliar $[r(\mathrm{~L})=-0.35, \mathrm{P}<0.0001$;
$r(\mathrm{~A})=0.38, \mathrm{P}<0.0001]$, y el diámetro de la cicatriz de la nuez $[r(\mathrm{~L})=-0.37, \mathrm{P}<0.0001 ; r(\mathrm{~A})=0.32, \mathrm{P}<0.0001]$ tuvieron una correlación negativa con la latitud y positiva con la altitud; mientras el número de estomas $/ \mathrm{cm}^{2}$ se correlacionó negativamente con la altitud $(r=-0.40$, $\mathrm{P}<0.0001)$. En cambio, los caracteres del fruto en $Q$. sartorii y $Q$. xalapensis no presentaron ninguna correlación significativa $(\mathrm{P}>0.06)$ con la altitud o la latitud.

$\mathrm{El}$ análisis de varianza detectó diferencias significativas entre poblaciones de las 2 especies para el largo de bellota $(\mathrm{F}=8.72, \mathrm{gl}=10, \mathrm{P}<0.0001, \mathrm{~N}=47)$, los diámetros de bellota $(\mathrm{F}=18.83, \mathrm{gl}=10, \mathrm{P}<0.0001, \mathrm{~N}=47)$, cúpula $(\mathrm{F}=66.30, \mathrm{gl}=10, \mathrm{P}<0.0001, \mathrm{~N}=47)$ y nuez $(\mathrm{F}$ $=8.30, \mathrm{gl}=10, \mathrm{P}<0.0001, \mathrm{~N}=47)$; así como el número de aréolas $/ \mathrm{mm}^{2}(\mathrm{~F}=18.34, \mathrm{gl}=10, \mathrm{P}<0.0001 \mathrm{~N}=47)$. Las diferencias entre especies se muestran en el gradiente latitudinal y altitudinal donde se colectaron las especies (Figs. 3, 4).

Los resultados de los análisis de similitud, donde se incluyó únicamente el material recolectado, separan 2 grupos. Uno se formó por los individuos de Quercus sartorii y el otro por los individuos de Q. xalapensis (Fig. 5). Los 2 grupos correspondientes a las especies se asociaron a un nivel de similitud cercano a 8.99, al cual se unió un individuo (43) de la población 11 de Tlahualompa, Hidalgo, separándose del resto debido a que presentó un mayor número de aréolas. Los caracteres cualitativos que contribuyeron a la separación de los grupos (especies) en el análisis de similitud son la forma de las aréolas, la ramificación de las vénulas, el recorrido de la vena secundaria dentro del diente y la distribución de los vasos. En Q. sartorii el recorrido de la vena secundaria dentro del diente es excéntrico, la forma de las aréolas es cuadrangular o poligonal (Fig. 6a, c), las vénulas generalmente no se ramifican, la felodermis está escasamente desarrollada o ausente (Fig. 7a) y la distribución de vasos se presentó en hileras radiales (Fig. 7c). Por el contrario, en Q. xalapensis el recorrido de la vena secundaria dentro del diente es central, las aréolas son de forma irregular (Fig. 6b, d), las vénulas se ramifican 1 o 2 veces, la felodermis se compone de varios estratos de células (Fig. 7b) y los vasos no se presentaron en hileras radiales (Fig. 7d). Los caracteres que permiten separar las especies estudiadas se resumen en el Cuadro 2.

El fenograma que incluye a los 164 individuos mostró que no hay grupos constituidos exclusivamente por individuos de Quercus sartorii o de Q. xalapensis (Fig. 8). Los individuos de las 2 especies se asociaron indistintamente en más de 10 subgrupos, en su mayoría de poblaciones de distribución alopátrica y carentes de frutos. La amplitud presente en los niveles de agrupamiento osciló entre 0.73 y 14.9 . 

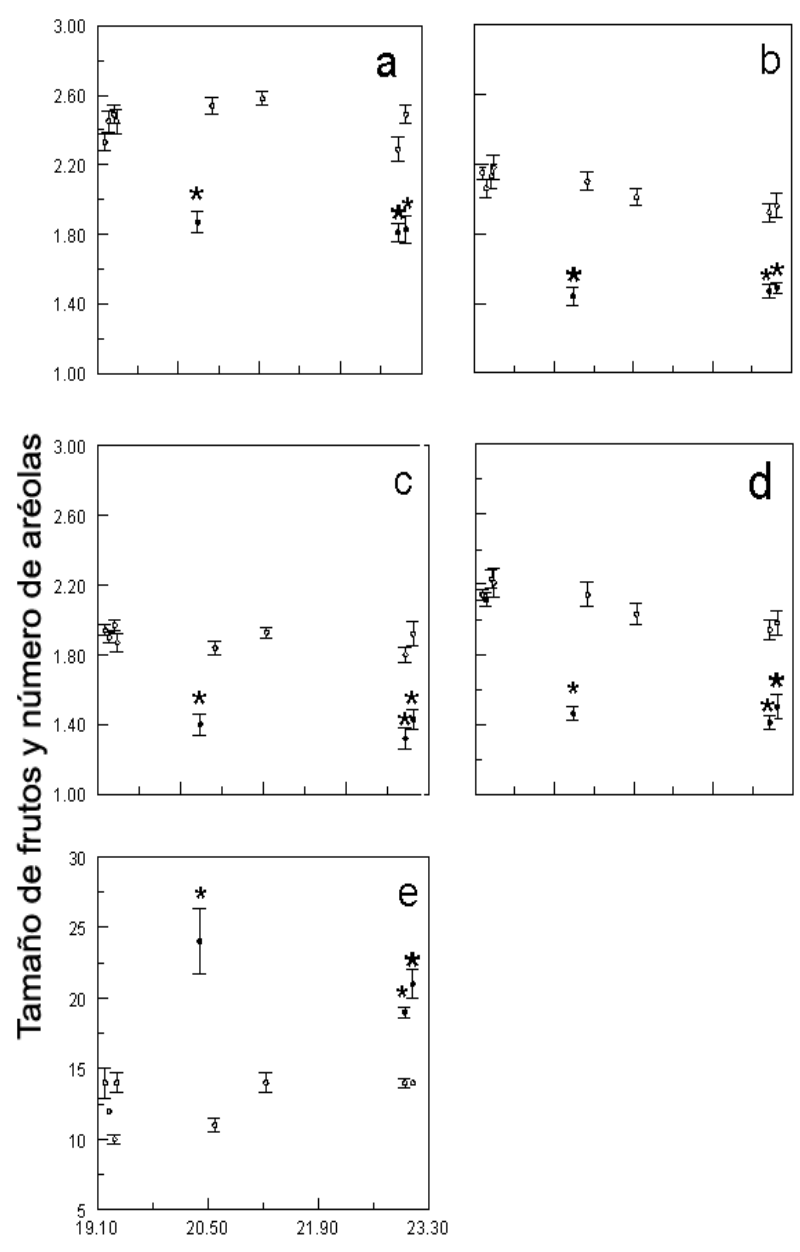

Latitud

Figura 3. Diferencias significativas para la longitud de la bellota (a), diámetro de la bellota (b), diámetro de la nuez (c), diámetro de la cúpula (d) y el número de aréolas (e) entre poblaciones de Quercus sartorii y Q.xalapensis. El circulo representa la media y la línea la desviación estándar para cada población. Circulo lleno $=Q$. sartorii, circulo vacío $=Q$. xalapensis. Asteriscos indican diferencias significativas $(\mathrm{P}<0.05$, Tukey) entre especies.
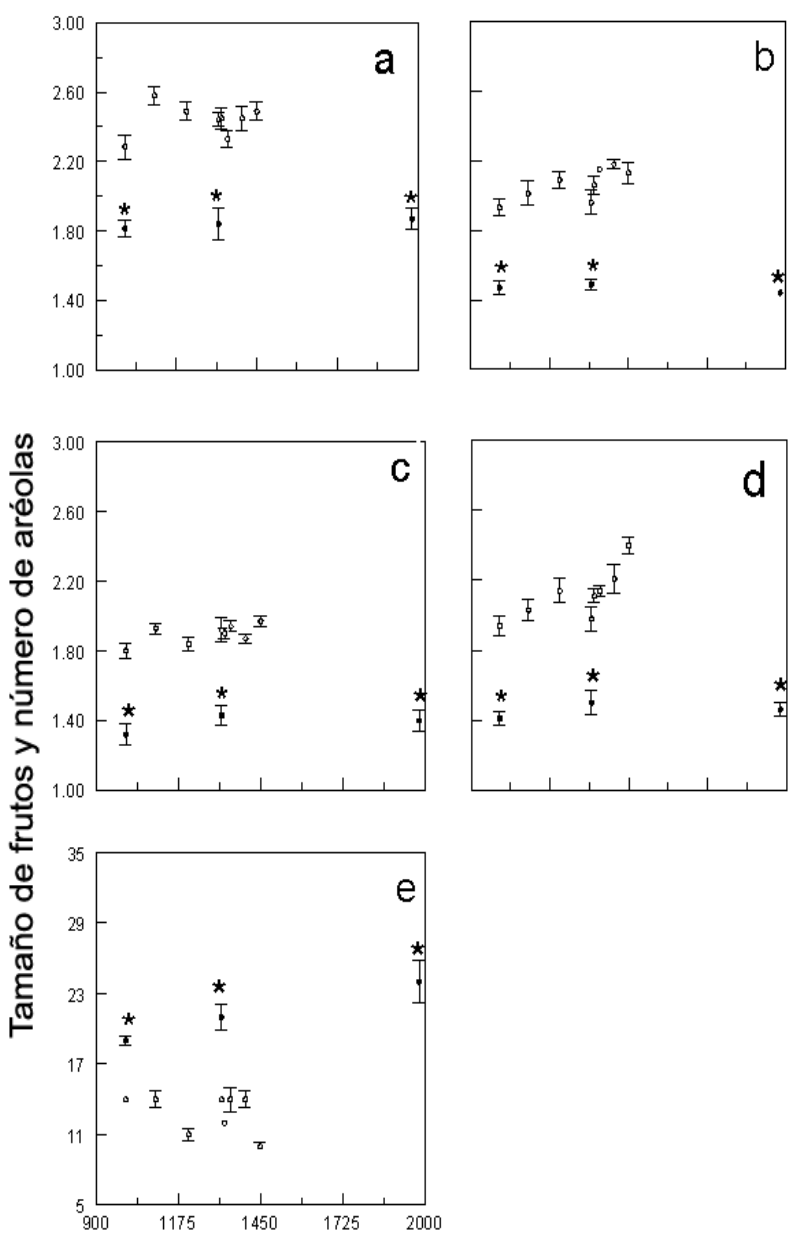

\section{Altitud (m)}

Figura 4. Diferencias significativas para la longitud de la bellota (a), diámetro de la bellota (b), diámetro de la nuez (c), diámetro de la cúpula (d) y el número de aréolas (e) entre poblaciones de Quercus sartorii y Q. xalapensis. El círculo representa la media y la línea la desviación estándar para cada población. Círculo lleno $=Q$. sartorii, circulo vacío $=Q$. xalapensis. Asteriscos indican diferencias significativas $(\mathrm{P}<0.05$, Tukey) entre especies. 

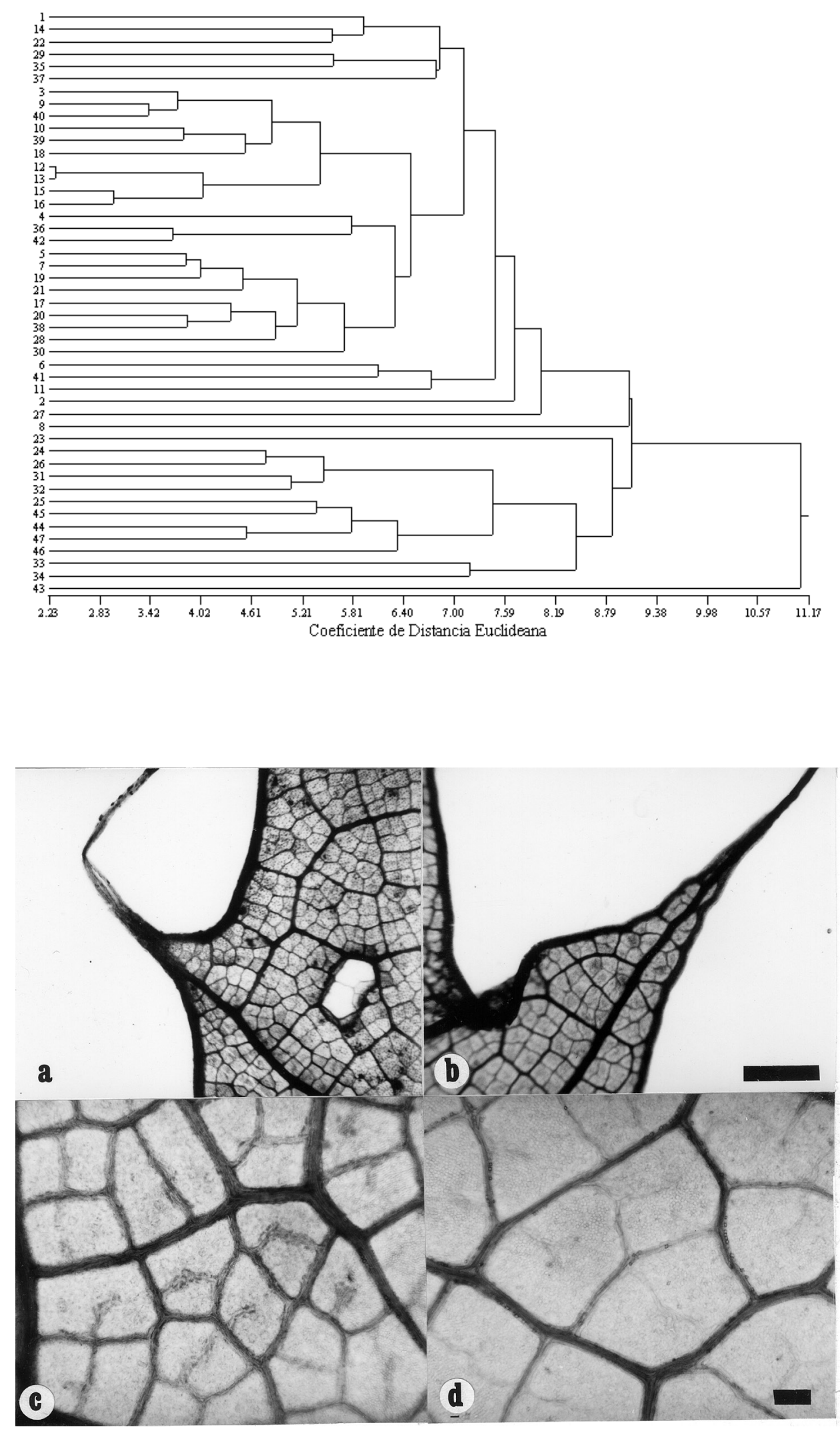

Figura 5. Fenograma para 47 individuos recolectados de las 11 poblaciones de Quercus sartorii y $Q$. xalapensis generado con el coeficiente de distancia euclideana y UPGMA. La matriz se puede consultar por solicitud.

Figura 6. Detalles del patrón de venación de Quercus sartorii y $Q$. xalapensis. a-b. Recorrido de la vena al diente. a, Quercus sartorii, Martínez 160; b, Q. xalapensis, Martínez 120. c-d. Aréolas. c, $Q$. sartorii, Martinez 158; d, Q. xalapensis, Martínez 119. Escala: a-b, $1 \mathrm{~mm}$; c-d, $100 \mu \mathrm{m}$. 


\section{Discusión}

Los análisis multivariados y el análisis de similitud mostraron que los caracteres que contribuyeron a la delimitación de Quercus sartorii y $Q$. xalapensis fueron el diámetro de la bellota, el diámetro de la nuez y el diámetro de la cúpula. La bellota fue de mayor tamaño en $Q$. xalapensis que en $Q$. sartorii. La longitud y diámetro de la bellota fueron considerados importantes por Trelease (1924) para diferenciar ambas especies. Otros caracteres, como el largo de la bellota y de la nuez, se compartieron en 3 individuos de las poblaciones simpátricas de Tamaulipas, lo que podría sugerir una posible hibridación entre especies. Es recomendable realizar estudios con marcadores moleculares en dichas poblaciones simpátricas para confirmar el flujo de genes, como recientemente se demostró para otras especies de encinos (Albarrán-Lara et al., 2010; Peñaloza-Ramírez et al., 2010). Los resultados del análisis de varianza mostraron que la variación de los caracteres del fruto es significativa $(\mathrm{P}<0.05)$ aún en las poblaciones simpátricas. El análisis de correlación mostró que la variación interespecífica de estos caracteres del fruto no está correlacionada con el gradiente altitudinal ni latitudinal en el que se recolectaron las poblaciones de ambos taxa. Al menos para los caracteres del fruto, no existió una variación clinal como la detectada para otras especies (Aizen y Woodcock, 1992).

El análisis de similitud, en el cual se incluyó exclusivamente el material recolectado en campo, confirmó los resultados de los análisis multivariados. El fenograma mostró 2 grupos, uno que incluye a todos los individuos de Quercus sartorii y otro a los de Q. xalapensis. Este análisis evidenció que en los grupos de cada especie no hubo formación de subgrupos que asociaran exclusivamente individuos de la misma población, sino que éstos se formaron por la asociación de individuos de diferentes poblaciones. Es decir, existe mayor similitud entre individuos de diferentes poblaciones que entre los individuos de una misma población. Este comportamiento sugiere que la variación morfológica no responde a un gradiente altitudinal ni a uno latitudinal (Sneath y Sokal, 1973). Los resultados de este análisis no apoyan la existencia de las formas $Q$. xalapensis f. jalapae y Q. xalapensis f. surculina propuestas por Trelease (1924) para Q. xalapensis, confirmando lo señalado por Valencia (2004).

Cuadro 2. Caracteres morfológicos y anatómicos diagnósticos en la delimitación taxonómica de Quercus sartorii y Q. xalapensis. En los caracteres cuantitativos se presenta la media \pm error estándar y entre paréntesis el valor mínimo y máximo

\begin{tabular}{|c|c|c|}
\hline Carácter & Quercus sartorii & Quercus xalapensis \\
\hline Diámetro de la bellota (límite entre la nuez y la cúpula, mm) & $1.37 \pm 0.1(1.2-1.5)$ & $2.0 \pm 0.16(1.8-2.5)$ \\
\hline Diámetro de la nuez (mm) & $1.2 \pm 0.11(1.0-1.4)$ & $1.9 \pm 0.11(1.7-2.2)$ \\
\hline Diámetro de la cúpula $(\mathrm{mm})$ & $1.4 \pm 0.11(1.2-1.6)$ & $2.0 \pm 0.17(1.8-2.6)$ \\
\hline Número de aréolas $\left(\mathrm{cm}^{2}\right)$ & $23 \pm 6(18-34)$ & $12 \pm 2.2(9-19)$ \\
\hline Recorrido de la vena secundaria dentro del diente de la hoja & Excéntrico & Central \\
\hline Forma de las aréolas & Cuadrangular o poligonal & Irregular \\
\hline Ramificación de las vénulas & Ninguna o rara vez una & 1 o 2 veces \\
\hline Número de células de la felodermis & 1 a 3 & 4 a 7 \\
\hline Distribución de los vasos & En hileras radiales & En diagonal \\
\hline
\end{tabular}




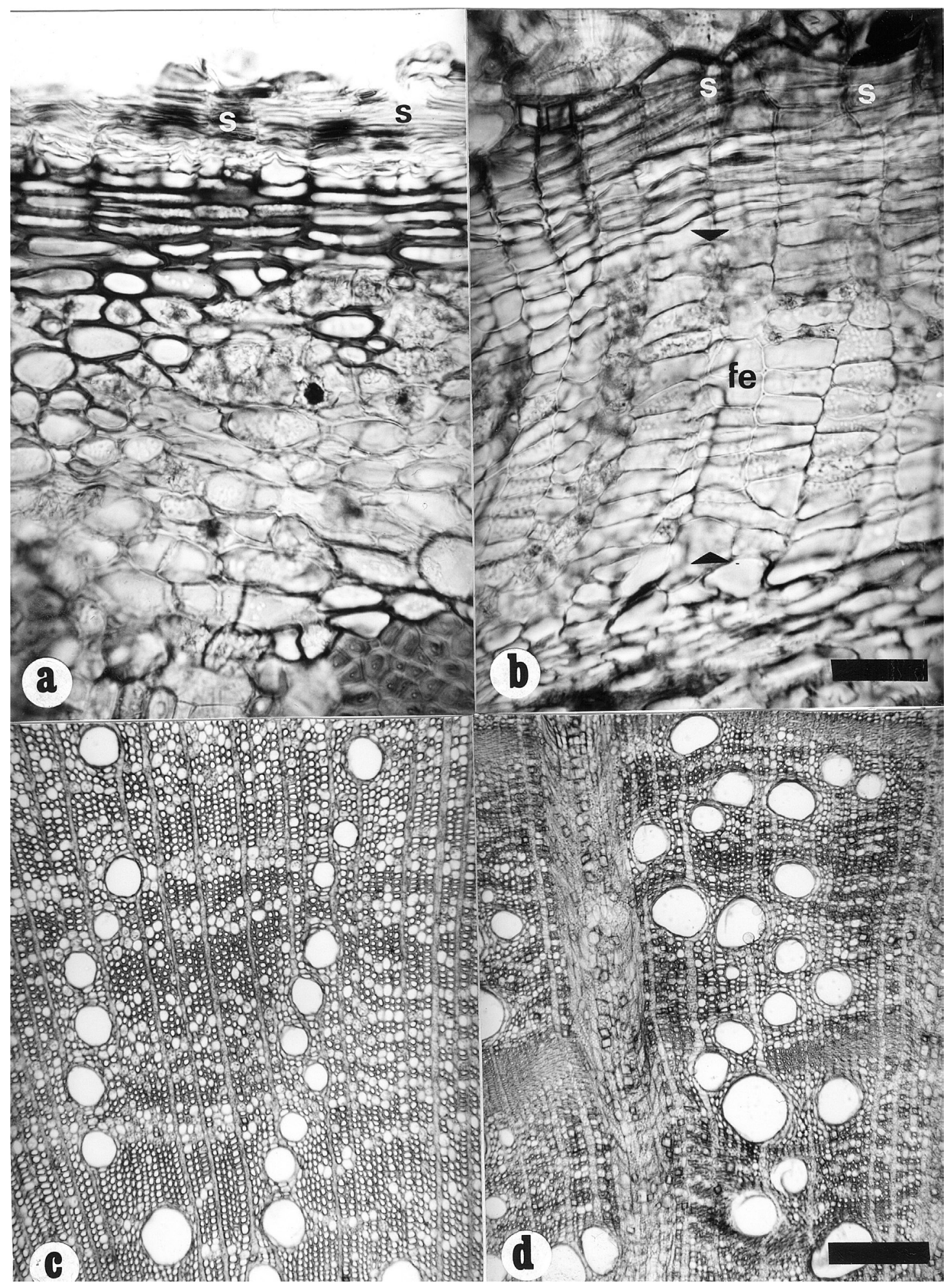

Figura 7. Corteza y madera de Quercus sartorii y Q. xalapensis, cortes transversales. a-b. Detalles de peridermis; las flechas señalan a las células de la felodermis. a, Quercus sartorii, Martínez 161; b, Q. xalapensis, Martínez 130. c-d. Distribución de vasos en la madera. c, Q. sartorii, Martínez 159; d, Q. xalapensis, Martínez 120. Escala: a-b, $50 \mu \mathrm{m}$; c-d, $200 \mu \mathrm{m}$; s, súber; fe, felodermis. 


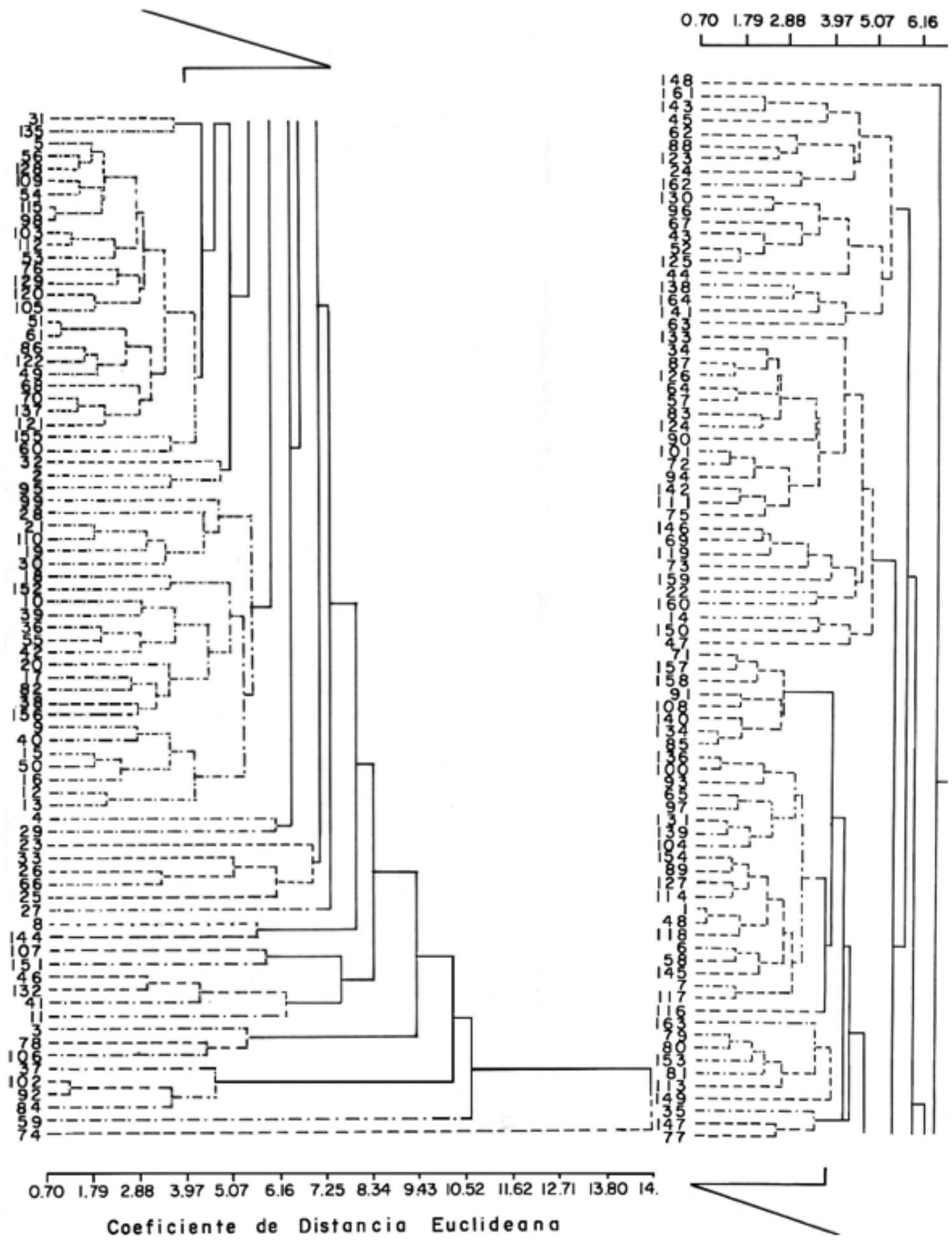

Figura 8. Fenograma para 164 individuos de Quercus sartorii y Q. xalapensis generado con el coeficiente de distancia Euclideana y UPGMA. La matriz se puede consultar por solicitud. 
La importancia del fruto en los trabajos taxonómicos del género Quercus ha sido resaltada por varios autores (Muller y McVaugh, 1972; Zavala, 1999; Cristofolini y Crema, 2005). Zavala (1999) señala que la morfología de las bellotas de encinos se ha usado principalmente cuando se analizan individuos de una especie o entre especies cercanamente emparentadas. En el caso de las especies estudiadas, el diámetro de la bellota no se yuxtapone entre ellas (Cuadro 2). Sin embargo, son escasos los trabajos que han estudiado los caracteres del fruto, porque los ejemplares de herbario carecen de ellos, o bien los presentan inmaduros. Los caracteres vegetativos con los que tradicionalmente se han identificado estas especies (largo de la lámina foliar y del pecíolo) resultaron importantes solamente en el segundo y tercer componente en el ACP. El análisis de varianza mostró que dichos caracteres presentaron una amplia variación intra e interespecífica que no permitió reconocer como entidades diferentes a $Q$. sartorii de $Q$. xalapensis conforme se ha detectado en otras especies de Quercus y otros géneros (Muller y McVaugh, 1972; Mayer, 1991; Tyteca y Dufrêne, 1994; GonzálezRodríguez y Oyama 2005; Albarrán Lara et al., 2010). Más aún, lo anterior se resalta en los resultados del análisis de similitud que incluye los individuos provenientes de herbarios que en su mayoría son estériles y por ende, los atributos vegetativos no permiten distinguir entre especies.

Los resultados obtenidos con los caracteres macromorfológicos mostraron que ambas especies comparten la forma y tamaño de hoja, el tipo de margen, el largo y el ancho de pecíolo y el número de dientes. Esto confirma lo mencionado por Jones (1986), Muller y McVaugh (1972) y Zavala (1999), quienes señalan que las hojas de los encinos presentan una amplia variación morfológica y sólo en muy pocas excepciones se presenta una morfología constante. $\mathrm{Al}$ incluir los caracteres de la arquitectura foliar señalados por Martínez-Cabrera et al. (2003) en el análisis de similitud, encontramos que el recorrido de la vena secundaria dentro del diente, la forma y el número de aréolas, así como la ramificación de las vénulas, contribuyeron en la separación de las especies estudiadas, confirmando que el patrón de venación provee caracteres útiles en la delimitación de especies de Quercus (Valencia y Delgado, 1995). El tipo de diente que presentan ambas especies es aristado, este carácter coincide con lo que Hickey y Wolfe (1975) describieron para el orden Fagales. Sin embargo, el recorrido que tiene la vena secundaria dentro del diente fue diferente en las especies estudiadas (Martínez-Cabrera et al., 2003). En $Q$. sartorii el recorrido de la vena es excéntrico, mientras que en $Q$.xalapensis es central. Este carácter y, en general, la morfología del diente, se considera de valor diagnóstico en los trabajos taxonómicos (Hickey y Wolfe, 1975).

El análisis de similitud confirmó que el número de célu- las de la felodermis y la distribución de vasos en la madera también son importantes en la separación de Quercus sartorii y $Q$. xalapensis, como sugieren Martínez-Cabrera et al. (2003). El número de células de la felodermis fue contrastante entre ambas especies y ayudó a la delimitación taxonómica. La felodermis junto con algunos atributos del floema como esclerénquima y dilatación de los radios presentan valor diagnóstico (Roth, 1981). El arreglo de los vasos en la madera en $Q$. sartorii fue en hileras radiales largas y en $Q$. xalapensis fue en diagonal. La distribución de los vasos en hileras radiales concuerda con las descripciones de varias especies de Quercus como $Q$. convallata Trel., Q. obtusata Humb. et Bonpl., Q. crassifolia Humb. et Bonpl., Q. rugosa Née y $Q$. candicans Née (de la Paz Pérez 1976, 1982).

Los resultados apoyan el reconocimiento de Quercus sartorii y $Q$. xalapensis propuesto por Trelease (1924) y Valencia (2004). En consecuencia, sugerimos que los caracteres del diámetro del fruto sean considerados como críticos para la delimitación taxonómica de dichas especies, ya que presentaron una variación discreta que permitió separarlas inclusive en condiciones de simpatría. Por último, sería importante incluir otras poblaciones más sureñas (Chiapas) de Q. xalapensis con la finalidad de confirmar que las diferencias encontradas entre las especies estudiadas se mantienen.

\section{Agradecimientos}

A CONACyT, por la beca (Núm. 159282) otorgada a la primera autora para realizar estudios de posgrado; a los herbarios CHAP, CHAPA, ENCB, MEXU, MO, NY y XAL, por las facilidades para tener acceso al material consultado y por la oportunidad de remover material para el estudio del patrón de venación, y a Héctor Hernández, por el trabajo fotográfico de cuarto obscuro.

\section{Literatura citada}

Aizen, M. A. y H. Woodcock. 1992. Latitudinal trends in acorn size and geographical range in the North American oaks. Journal of Biogeography 17:327-332.

Albarrán-Lara, A. L., L. Mendoza-Cuenca, S. Valencia-Ávalos, A. González-Rodríguez y K. Oyama. 2010. Leaf fluctuating asymmetry increases with hybridization and introgression between Quercus magnoliifolia and Quercus resinosa (Fagaceae) through an altitudinal gradient in Mexico. International Journal of Plant Science 171:310-322.

Cristofolini, G. y S. Crema. 2005. A morphometric study of 
the Quercus crenata species complex. Botanica Helvetica 115:155-167.

De la Paz Pérez O., C. 1974. Anatomía de la madera de cinco especies de encinos de Durango. Boletín Técnico del Instituto Nacional de Investigaciones Forestales (México) 43:1-75.

De La Paz Pérez O., C. 1976. Características anatómicas de cinco encinos de México. Boletín Técnico del Instituto Nacional de Investigaciones Forestales (México) 46:1-43.

De La Paz Pérez O., C. 1982. Estructura anatómica de cinco especies del género Quercus. Boletín Técnico del Instituto Nacional de Investigaciones Forestales (México) 43:1-63.

González-Rodríguez, A. y K. Oyama. 2005. Leaf morphometric variation in Quercus affinis and Q. laurina (Fagaceae), two hybridizing Mexican red oaks. Botanical Journal of the Linnean Society 147:427-435.

Hickey, L. y J. A. Wolfe. 1975. The bases of angiosperm phylogeny: vegetative morphology. Annals of the Missouri Botanical Garden 62:538-589.

Jones, H. J. 1986. Evolution of the Fagaceae: the implications of foliar features. Annals of the Missouri Botanical Garden 73:228-275.

Jensen, R. J., S. C. Hokanson, J. G. Isebrands y J. F. Hancock. 1993. Morphometric variation in oaks of the Apostle Islands in Wisconsin: evidence of hybridization between Quercus rubra and Q. ellipsoidalis (Fagaceae). American Journal of Botany 80:1358-1366.

Johnson, R. A. y D. W. Wichern. 1982. Applied multivariate statistical analysis. Prentice Hall, Englewood Cliffs, New Jersey. $816 \mathrm{p}$.

Manos, P.S., J. J. Doyle y K. C. Nixon. 1999. Phylogeny, biogeography, and processes of molecular differentiation in Quercus subgenus Quercus (Fagaceae). Molecular Phylogenetics and Evolution 12:333-349.

Martínez-Cabrera, D., T. Terrazas y F. Zavala 2003. Arquitectura foliar y anatomía de la corteza y madera de Quercus sartorii y Q. xalapensis (Fagaceae). Boletín de la Sociedad Botánica de México 73:63-72.

Mayer, S. S. 1991. Morphological variation in hawaiian Wikstroemia (Thymelaeaceae). Systematic Botany 18:248260.

Muller, C. y R. McVaugh. 1972. The oaks (Quercus) described by Née (1801), and by Humboldt \& Bonpland (1809), with comments on related species. Contributions from the University of Michigan Herbarium 7:507-522.

Neff, A. N. y L. F. Marcus. 1980. A survey of multivariate methods for systematics. New York. 57 p.

Nixon, K. C. 1997. Fagaceae. Flora of North America, North of Mexico, vol. 3, Magnoliophyta: Magnoliidae and Hamamelidae. Oxford University Press, New York. p. 436-506.

Nixon, K. C. y C. H. Muller. 1992. The taxonomic resurrection of Quercus laceyi Small (Fagaceae). Sida 15:57-69.
Nixon, K. C. y C. H. Muller 1993. The Quercus hypoxantha complex in northeastern México. Brittonia 45:146-153.

Palmer, E. J. 1948. Hybrid oaks of North America. Journal of the Arnold Arboretum 29:1-48.

Peñaloza-Ramírez, J. M., A. González-Rodríguez, L. MendozaCuenca, H. Caron, A. Kremer y K. Oyama. 2010. Interspecific gene flow in a multispecies oak hybrid zone in the Sierra Tarahumara of Mexico Annals of Botany 105:389-399.

Rohlf, F. J. 1997. NTSYSpc. Numerical taxonomy and multivariate analysis system, ver. 2.0. Applied Biostatistics, New York. 200 p.

Romero, R. S. 2006. Revisión taxonómica del complejo Acutifoliae de Quercus (Fagaceae) con énfasis en su representación en México. Acta Botanica Mexicana 76:1-45.

Roth, I. 1981. Structural patterns of tropical barks. Encyclopedia of plant anatomy. Gebruder Borntraeger, Berlin. 609 p.

Rzedowski, J. 1978. Vegetación de México. Limusa, México, D.F. 432 p.

SAS, Institute. 1989. SAS Procedures Guide Version 6.04. SAS Institute, Cary, North Carolina. 1028 p.

Sneath, P. H. A. 1976. Phenetic taxonomy at the species level and above. Taxon 25:437-450.

Sneath, P. H. A. y R. R. Sokal. 1973. Numerical taxonomy. The principles and practices of numerical classification. Freeman, San Francisco, California. 573 p.

Trelease, W. 1924. The American oaks. Memoirs of the National Academy of Science. Government Printing Office, Washington, D.C. 255 p.

Tyteca, D. y M. Dufrêne. 1994. Biostatistical studies of western European allogamus populations of the Epipactus helleborine (L.) Crantz species group (Orchidaceae). Systematic Botany 19:422-442.

Valencia, A. S. 2004. Diversidad del género Quercus (Fagaceae) en México. Boletín de la Sociedad Botánica de México 75:33-53.

Valencia, A. S. y S. A. Delgado. 1995. Arquitectura de hojas como una herramienta taxonómica en el reconocimiento de individuos de encinos pertenecientes a Quercus affinis Scheid y Q. laurina Humboldt \& Bonpland. Memorias del III seminario nacional sobre utilización de encinos, tomo II; 4-6 de noviembre de 1992, J. S. Marroquín de la F. (ed.). Universidad Autónoma de Nuevo León, Linares. p. 850-859.

Vázquez, M. L., S. Valencia-A. y K. C. Nixon 2004. Notes on red oaks (Quercus sect. Lobatae) in eastern México with description of a new species. Quercus hirtiifolia. Brittonia 56:136-142.

Zar, J. 1999. Biostatistical analysis. Prentice Hall, Englewood Cliffs, New Jersey. 663 p.

Zavala C., F. 1999. Variabilidad y riqueza de los encinos de México. Chapingo, Serie Ciencias Forestales y del Ambiente 5:113-122. 
Apéndice 1. Material de Quercus sartorii (sar) y Q. xalapensis (xal) recolectado en campo (C) o procedente de herbario (H) que se incluyó en los diferentes análisis ( $\mathrm{CP}$, componentes principales; $\mathrm{D}$, discriminante; $\mathrm{V}$, varianza, $\mathrm{S}$, similitud) identificado con un número de referencia con su correspondiente localidad y colector.

\begin{tabular}{|c|c|c|c|c|c|}
\hline Taxon & Material & Análisis & $\begin{array}{l}\text { Núm.de } \\
\text { referencia }\end{array}$ & Localidad & Colector \\
\hline sar & $\mathrm{C}$ & $\begin{array}{l}\text { CP, D, } \\
\mathrm{V}, \mathrm{S}\end{array}$ & $1-3$ & $\begin{array}{l}\text { Veracruz, Mpio. Xico, } 1350 \mathrm{~m}, \\
19^{\circ} 20^{\prime} \mathrm{N}, 96^{\circ} 57^{\prime} \mathrm{O}\end{array}$ & D. Martínez y F. Zavala 116-118 (CHAPA) \\
\hline$x a l$ & $\mathrm{C}$ & $\begin{array}{l}\text { CP, D, } \\
\text { V, S }\end{array}$ & $4-8$ & $\begin{array}{l}\text { Veracruz, Mpio. Xalapa, } 1325 \text { m, } \\
19^{\circ} 30^{\prime} \mathrm{N}, 96^{\circ} 56^{\prime} \mathrm{O}\end{array}$ & D. Martinez 119-123 (CHAPA) \\
\hline$x a l$ & $\mathrm{C}$ & $\begin{array}{l}\text { CP, D, } \\
\text { V, S }\end{array}$ & $9-13$ & $\begin{array}{l}\text { Veracruz, Mpio. San Andrés Tlalnehuayocan, } \\
1450 \mathrm{~m}, 19^{\circ} 32^{\prime} \mathrm{N}, 96^{\circ} 59^{\prime} \mathrm{O}\end{array}$ & D. Martínez 124-128 (CHAPA) \\
\hline$x a l$ & $\mathrm{C}$ & $\begin{array}{l}\text { CP, D, } \\
\mathrm{V}, \mathrm{S}\end{array}$ & $14-18$ & $\begin{array}{l}\text { Veracruz, Mpio Banderilla, } \\
1400 \mathrm{~m}, 19^{\circ} 34^{\prime} \mathrm{N}, 96^{\circ} 56^{\prime} \mathrm{O}\end{array}$ & D. Martínez 129-133 (CHAPA) \\
\hline$x a l$ & $\mathrm{C}$ & $\begin{array}{l}\text { CP, D, } \\
\text { V, S }\end{array}$ & $19-22$ & $\begin{array}{l}\text { Tamaulipas, Mpio. Gómez Farías, } \\
1000 \mathrm{~m}, 23^{\circ} 00^{\prime} \mathrm{N}, 99^{\circ} 14^{\prime} \mathrm{O}\end{array}$ & D. Martínez y F. Zavala 134-137 (CHAPA) \\
\hline sar & $\mathrm{C}$ & $\begin{array}{l}\text { CP, D, } \\
\text { V, S }\end{array}$ & $23-26$ & $\begin{array}{l}\text { Tamaulipas, Mpio. Gómez Farías, } \\
1000 \mathrm{~m}, 23^{\circ} 00^{\prime} \mathrm{N}, 99^{\circ} 14^{\prime} \mathrm{O}\end{array}$ & D. Martínez y F. Zavala 138-141 (CHAPA) \\
\hline$x a l$ & $\mathrm{C}$ & $\begin{array}{l}\text { CP, D, } \\
\text { V, S }\end{array}$ & $27-30$ & $\begin{array}{l}\text { Tamaulipas, Mpio. Gómez Farías, } \\
1320 \mathrm{~m}, 23^{\circ} 03^{\prime} \mathrm{N}, 99^{\circ} 15^{\prime} \mathrm{O}\end{array}$ & D. Martínez y F. Zavala 142-145 (CHAPA) \\
\hline sar & $\mathrm{C}$ & $\begin{array}{l}\mathrm{CP}, \mathrm{D} \\
\mathrm{V}, \mathrm{S}\end{array}$ & $31-34$ & $\begin{array}{l}\text { Tamaulipas, Mpio. Gómez Farías, } \\
1320 \mathrm{~m}, 23^{\circ} 03^{\prime} \mathrm{N}, 99^{\circ} 15^{\prime} \mathrm{O}\end{array}$ & D. Martínez y F. Zavala 146-149 (CHAPA) \\
\hline$x a l$ & $\mathrm{C}$ & $\begin{array}{l}\text { CP, D, } \\
\text { V, S }\end{array}$ & $35-37$ & $\begin{array}{l}\text { San Luis Potosí, Mpio. Xilitla, } \\
1100 \mathrm{~m}, 21^{\circ} 24^{\prime} \mathrm{N}, 99^{\circ} 00^{\prime} \mathrm{O}\end{array}$ & D. Martínez y F. Zavala 150-152 (CHAPA) \\
\hline$x a l$ & $\mathrm{C}$ & $\begin{array}{l}\text { CP, D, } \\
\text { V, S }\end{array}$ & $38-42$ & $\begin{array}{l}\text { Hidalgo, Mpio. Tlanchinol, } \\
1210 \mathrm{~m}, 20^{\circ} 59^{\prime} \mathrm{N}, 98^{\circ} 39^{\prime} \mathrm{O}\end{array}$ & D. Martinez 153-157 (CHAPA) \\
\hline sar & $\mathrm{C}$ & $\begin{array}{l}\text { CP, D, } \\
\text { V, S }\end{array}$ & $43-47$ & $\begin{array}{l}\text { Hidalgo, Mpio. Zacualtipán, } \\
1980 \text { m, } 20^{\circ} 39^{\prime} \mathrm{N}, 98^{\circ} 40^{\prime} \mathrm{O}\end{array}$ & D. Martinez 158-162 (CHAPA) \\
\hline$x a l$ & $\mathrm{H}$ & S & 48 & Chiapas, Mpio. Motozintla de Mendoza & D. E. Breedlove 42675 (ENCB) \\
\hline$x a l$ & $\mathrm{H}$ & S & 49 & Hidalgo, Mpio. Acaxochitlán & A. Villa $286(\mathrm{ENCB})$ \\
\hline sar & $\mathrm{H}$ & $\mathrm{S}$ & 50 & Hidalgo, Mpio. Actopan & L. González Quintero 2164 (ENCB) \\
\hline sar & $\mathrm{H}$ & $\mathrm{S}$ & 51,52 & Hidalgo, Mpio. Chapulhuacán & J. Rzedowski 23167, 23168 (ENCB) \\
\hline$x a l$ & $\mathrm{H}$ & $\mathrm{S}$ & 53,54 & Hidalgo, Mpio. Lolotla & L. Vela 226 (ENCB), S. Ochoa 937 (ENCB) \\
\hline sar & $\mathrm{H}$ & $\mathrm{S}$ & 55,56 & Hidalgo, Mpio. Molango & S. Ochoa 920 (ENCB), L. González 1600 \\
\hline
\end{tabular}

(ENCB) 
Apéndice 1. Continúa.

\begin{tabular}{|c|c|c|c|c|c|}
\hline Taxon & Material & Análisis & $\begin{array}{l}\text { Núm.de } \\
\text { referencia }\end{array}$ & Localidad & Colector \\
\hline sar & $\mathrm{C}$ & $\begin{array}{l}\mathrm{CP}, \mathrm{D} \\
\mathrm{V}, \mathrm{S}\end{array}$ & $1-3$ & $\begin{array}{l}\text { Veracruz, Mpio. Xico, } 1350 \mathrm{~m}, \\
19^{\circ} 20^{\prime} \mathrm{N}, 96^{\circ} 57^{\prime} \mathrm{O}\end{array}$ & D. Martínez y F. Zavala 116-118 (CHAPA) \\
\hline$x a l$ & $\mathrm{C}$ & $\begin{array}{l}\text { CP, D, } \\
\mathrm{V}, \mathrm{S}\end{array}$ & $4-8$ & $\begin{array}{l}\text { Veracruz, Mpio. Xalapa, } 1325 \text { m, } \\
19^{\circ} 30^{\prime} \mathrm{N}, 96^{\circ} 56^{\prime} \mathrm{O}\end{array}$ & D. Martínez 119-123 (CHAPA) \\
\hline$x a l$ & $\mathrm{C}$ & $\begin{array}{l}\text { CP, D, } \\
\text { V, S }\end{array}$ & $9-13$ & $\begin{array}{l}\text { Veracruz, Mpio. San Andrés Tlalnehuayocan, } \\
1450 \mathrm{~m}, 19^{\circ} 32^{\prime} \mathrm{N}, 96^{\circ} 59^{\prime} \mathrm{O}\end{array}$ & D. Martinez 124-128 (CHAPA) \\
\hline$x a l$ & $\mathrm{C}$ & $\begin{array}{l}\text { CP, D, } \\
\text { V, S }\end{array}$ & $14-18$ & $\begin{array}{l}\text { Veracruz, Mpio Banderilla, } \\
1400 \mathrm{~m}, 19^{\circ} 34^{\prime} \mathrm{N}, 96^{\circ} 56^{\prime} \mathrm{O}\end{array}$ & D. Martínez 129-133 (CHAPA) \\
\hline$x a l$ & $\mathrm{C}$ & $\begin{array}{l}\text { CP, D, } \\
\text { V, S }\end{array}$ & $19-22$ & $\begin{array}{l}\text { Tamaulipas, Mpio. Gómez Farías, } \\
1000 \mathrm{~m}, 23^{\circ} 00^{\prime} \mathrm{N}, 99^{\circ} 14^{\prime} \mathrm{O}\end{array}$ & D. Martínez y F. Zavala 134-137 (CHAPA) \\
\hline sar & $\mathrm{C}$ & $\begin{array}{l}\text { CP, D, } \\
\mathrm{V}, \mathrm{S}\end{array}$ & $23-26$ & $\begin{array}{l}\text { Tamaulipas, Mpio. Gómez Farías, } \\
1000 \mathrm{~m}, 23^{\circ} 00^{\prime} \mathrm{N}, 99^{\circ} 14^{\prime} \mathrm{O}\end{array}$ & D. Martínez y F. Zavala 138-141 (CHAPA) \\
\hline$x a l$ & $\mathrm{C}$ & $\begin{array}{l}\text { CP, D, } \\
\text { V, S }\end{array}$ & $27-30$ & $\begin{array}{l}\text { Tamaulipas, Mpio. Gómez Farías, } \\
1320 \mathrm{~m}, 23^{\circ} 03^{\prime} \mathrm{N}, 99^{\circ} 15^{\prime} \mathrm{O}\end{array}$ & D. Martínez y F. Zavala 142-145 (CHAPA) \\
\hline sar & $\mathrm{C}$ & $\begin{array}{l}\text { CP, D, } \\
\text { V, S }\end{array}$ & $31-34$ & $\begin{array}{l}\text { Tamaulipas, Mpio. Gómez Farías, } \\
1320 \mathrm{~m}, 23^{\circ} 03^{\prime} \mathrm{N}, 99^{\circ} 15^{\prime} \mathrm{O}\end{array}$ & D. Martínez y F. Zavala 146-149 (CHAPA) \\
\hline$x a l$ & $\mathrm{C}$ & $\begin{array}{l}\text { CP, D, } \\
\text { V, S }\end{array}$ & $35-37$ & $\begin{array}{l}\text { San Luis Potosí, Mpio. Xilitla, } \\
1100 \mathrm{~m}, 21^{\circ} 24^{\prime} \mathrm{N}, 99^{\circ} 00^{\prime} \mathrm{O}\end{array}$ & D. Martínez y F. Zavala 150-152 (CHAPA) \\
\hline$x a l$ & $\mathrm{C}$ & $\begin{array}{l}\text { CP, D, } \\
\text { V, S }\end{array}$ & $38-42$ & $\begin{array}{l}\text { Hidalgo, Mpio. Tlanchinol, } \\
1210 \mathrm{~m}, 20^{\circ} 59^{\prime} \mathrm{N}, 98^{\circ} 39^{\prime} \mathrm{O}\end{array}$ & D. Martínez 153-157 (CHAPA) \\
\hline sar & $\mathrm{C}$ & $\begin{array}{l}\text { CP, D, } \\
\mathrm{V}, \mathrm{S}\end{array}$ & $43-47$ & $\begin{array}{l}\text { Hidalgo, Mpio. Zacualtipán, } \\
1980 \text { m, } 20^{\circ} 39^{\prime} \text { N, } 98^{\circ} 40^{\prime} \mathrm{O}\end{array}$ & D. Martínez 158-162 (CHAPA) \\
\hline$x a l$ & $\mathrm{H}$ & $\mathrm{S}$ & 48 & Chiapas, Mpio. Motozintla de Mendoza & D. E. Breedlove 42675 (ENCB) \\
\hline xal & $\mathrm{H}$ & $\mathrm{S}$ & 49 & Hidalgo, Mpio. Acaxochitlán & A. Villa 286 (ENCB) \\
\hline sar & $\mathrm{H}$ & $\mathrm{S}$ & 50 & Hidalgo, Mpio. Actopan & L. González Quintero 2164 (ENCB) \\
\hline sar & $\mathrm{H}$ & $\mathrm{S}$ & 51,52 & Hidalgo, Mpio. Chapulhuacán & J. Rzedowski 23167, 23168 (ENCB) \\
\hline$x a l$ & $\mathrm{H}$ & $\mathrm{S}$ & 53,54 & Hidalgo, Mpio. Lolotla & L. Vela 226 (ENCB), S. Ochoa 937 (ENCB) \\
\hline sar & $\mathrm{H}$ & $\mathrm{S}$ & 55,56 & Hidalgo, Mpio. Molango & S. Ochoa 920 (ENCB), L. González 1600 \\
\hline
\end{tabular}

(ENCB) 
Apéndice 1. Continúa.

\begin{tabular}{|c|c|c|c|c|c|}
\hline Taxon & Material & Análisis & $\begin{array}{l}\text { Núm.de } \\
\text { referencia }\end{array}$ & Localidad & Colector \\
\hline sar & $\mathrm{H}$ & $\mathrm{S}$ & 57 & Hidalgo, Mpio. Pacula & R. Hernández 3729 (MEXU) \\
\hline$x a l$ & $\mathrm{H}$ & $\mathrm{S}$ & 58 & Hidalgo, Mpio. Tlanchinol & J. Rzedowski 28678 (ENCB) \\
\hline xal & $\mathrm{H}$ & $\mathrm{S}$ & 59,60 & Hidalgo, Mpio. Tenango de Doria & $\begin{array}{l}\text { H. Puig } 4698 \text { (ENCB), R. Hernández } 3325 \\
\text { (ENCB) }\end{array}$ \\
\hline sar & $\mathrm{H}$ & $\mathrm{S}$ & $61-67$ & Hidalgo, Mpio. Zacualtipán & $\begin{array}{l}\text { J. Rzedowski } 28666 \text { (ENCB), J. López G. } \\
248 \text { (MEXU), J. López G. } 249 \text { (XAL), } \\
\text { E. Guizar } 414 \text { (MEXU), J. López G. } 163 \\
\text { (CHAP), J. Rzedowski y R. Madrigal } 29460 \\
\text { (ENCB), J. López G. } 523 \text { (CHAP) }\end{array}$ \\
\hline sar & $\mathrm{H}$ & $\mathrm{S}$ & $68-71$ & Hidalgo, Mpio. Zimapán & $\begin{array}{l}\text { R. Hernández } 3886 \text { (MEXU), R. } \\
\text { Hernández } 6563 \text { (XAL), H. Puig } 4413 \\
\text { (ENCB), R. Hernández } 6562 \text { (ENCB) }\end{array}$ \\
\hline sar & $\mathrm{H}$ & $\mathrm{S}$ & 72 & $\begin{array}{l}\text { Nuevo León, Mpio. Dos Puertos Vista } \\
\text { Hermosa }\end{array}$ & K. Roe 1893 (MEXU) \\
\hline sar & $\mathrm{H}$ & $\mathrm{S}$ & 73,74 & Nuevo León, Mpio. Potrero Redondo & M. de Rayones 1893 (MEXU) \\
\hline sar & $\mathrm{H}$ & $\mathrm{S}$ & 74 & Nuevo León, Mpio. Santiago & V. Valdez 945, 946 (ENCB) \\
\hline sar & $\mathrm{H}$ & $\mathrm{S}$ & 76 & Puebla, Mpio. Nauzontla & F. Simón y A. Colín 154 (ENCB) \\
\hline sar & $\mathrm{H}$ & $\mathrm{S}$ & 77 & Puebla, Mpio. Necaxa & M. S. Fonseca s.n. (ENCB) \\
\hline sar & $\mathrm{H}$ & $\mathrm{S}$ & 78 & Puebla, Mpio. Tetela de Ocampo & F. Simón y A. Colín 151 (ENCB) \\
\hline xal & $\mathrm{H}$ & $\mathrm{S}$ & $79-82$ & Puebla, Mpio. Xicotepec de Juárez & $\begin{array}{l}\text { L. González } 285 \text { (ENCB), J. Rzedowski } \\
\text { 17209, } 23390 \text { (ENCB), L. González } 170\end{array}$ \\
\hline sar & $\mathrm{H}$ & $\mathrm{S}$ & 83 & Puebla, Mpio. Zacapoaxtla & (ENCB) \\
\hline xal & $\mathrm{H}$ & $\mathrm{S}$ & 84 & Puebla, Mpio. Cuetzala del Progreso & M. Guzmán s.n. (ENCB) \\
\hline sar & $\mathrm{H}$ & $\mathrm{S}$ & 85,86 & Querétaro, Mpio. Landa & $\begin{array}{l}\text { H. Rubio } 1117 \text { (XAL) H. Puig } 3618 \\
\text { (ENCB) }\end{array}$ \\
\hline sar & $\mathrm{H}$ & $\mathrm{S}$ & 87 & Querétaro, Mpio. Tancoyol & H. Puig 4484 (ENCB). \\
\hline sar & $\mathrm{H}$ & $\mathrm{S}$ & $\begin{array}{c}88-90 \\
101\end{array}$ & San Luis Potosí, Mpio. Ciudad del Maíz & $\begin{array}{l}\text { H. Puig } 3421 \text { (ENCB), J. Rzedowski 11127, } \\
8336,8380 \text { (ENCB) }\end{array}$ \\
\hline sar & $\mathrm{H}$ & $\mathrm{S}$ & 91 & San Luis Potosí, Mpio. Los Hoyos & J. Rzedowski 9361 (ENCB) \\
\hline sar & H. & $\mathrm{S}$ & 92,103 & San Luis Potosí, Mpio. Santa Catarina & $\begin{array}{l}\text { H. Puig } 3665 \text { (ENCB), D. Q. Cavagnaro } \\
474 \text { (MEXU) }\end{array}$ \\
\hline
\end{tabular}


Apéndice 1. Continúa.

\begin{tabular}{|c|c|c|c|c|c|}
\hline Taxon & Material & Análisis & $\begin{array}{l}\text { Núm.de } \\
\text { referencia }\end{array}$ & Localidad & Colector \\
\hline sar & $\mathrm{H}$ & $\mathrm{S}$ & 93 & $\begin{array}{l}\text { San Luis Potosí, Mpio. San Nicolás de } \\
\text { Tolentino }\end{array}$ & J. Rzedowski 11316 (ENCB) \\
\hline sar & $\mathrm{H}$ & $\mathrm{S}$ & $94-96$ & San Luis Potosí, Mpio. Tamasopo & $\begin{array}{l}\text { H. Puig } 3645 \text { (ENCB), J. Rzedowski } 10628 \\
\text { (ENCB), J. Flamand } 8 \text { (ENCB) }\end{array}$ \\
\hline sar & $\mathrm{H}$ & $\mathrm{S}$ & 97 & San Luis Potosí, Mpio. Tamazunchale & J. Rzedowski 11093 (ENCB) \\
\hline xal & $\mathrm{H}$ & $\mathrm{S}$ & $98-100$ & San Luis Potosí, Mpio. Xilitla & J. Rzedowski 7185, 7249, 12386 (ENCB) \\
\hline sar & $\mathrm{H}$ & $\mathrm{S}$ & 102 & San Luis Potosí, Mpio. Río Verde & J. Rzedowski 1650 (ENCB) \\
\hline$x a l$ & $\mathrm{H}$ & $\mathrm{S}$ & 104 & San Luis Potosí, Mpio. Ciudad del Maíz & K. Roe 2307 (ENCB) \\
\hline$x a l$ & $\mathrm{H}$ & $\mathrm{S}$ & 105 & San Luis Potosí, Mpio. Guadalcazar & J. Rzedowski 4732 (ENCB) \\
\hline xal & $\mathrm{H}$ & $\mathrm{S}$ & 106 & San Luis Potosí, Mpio. Tamasopo & $\begin{array}{l}\text { L. M. González } 3418 \text { (ENCB), K. Roe } 2273 \\
\text { (ENCB) }\end{array}$ \\
\hline sar & $\mathrm{H}$ & $\mathrm{S}$ & 108 & San Luis Potosí, Mpio. de Guadalcazar & D. E. Breedlove 63307 (MEXU) \\
\hline sar & $\mathrm{H}$ & $\mathrm{S}$ & $109-117$ & Tamaulipas, Mpio. Gómez Farías & $\begin{array}{l}\text { J. Rodney } 328 \text { (ENCB), A. Gómez Pompa } \\
2014 \text { (MEXU), E. Hernández X. et al. } \\
50279 \text { (MEXU), J. Rodney } 511 \text { (ENCB), } \\
\text { P. S. Martín y C. Saravia S.n. (ENCB), J. } \\
\text { Rodney 292, } 522 \text { (ENCB), P. S. Martín y } \\
\text { C. Saravia } 1205 \text { (ENCB), J. Rodney } 429 \\
\text { (ENCB) }\end{array}$ \\
\hline sar & $\mathrm{H}$ & $\mathrm{S}$ & 118 & Tamaulipas, Mpio. Jaumave. & M. Castañeda 2760 (ENCB) \\
\hline xal & $\mathrm{H}$ & $\mathrm{S}$ & 119 & Tamaulipas, Mpio. Ocampo & D. E. Breedlove 63627 (MEXU) \\
\hline sar & $\mathrm{H}$ & $\mathrm{S}$ & 120 & Puebla, Mpio. Tetela de Ocampo & F. Simón y A. Colín 207 (MEXU) \\
\hline sar & $\mathrm{H}$ & $\mathrm{S}$ & 121 & Tamaulipas, Mpio. Jaumave & E. Hernández X. et al. 5828 (MEXU) \\
\hline sar & $\mathrm{H}$ & $\mathrm{S}$ & 122 & Tlaxcala, Mpio. Panotla & S. Cruz G. et al. 794 (CHAP) \\
\hline xal & $\mathrm{H}$ & $\mathrm{S}$ & 123,124 & Veracruz, Mpio. Banderilla & $\begin{array}{l}\text { T. C. Valdovinos } 5 \text { (ENCB), M. G. Zola } 531 \\
\text { (XAL) }\end{array}$ \\
\hline$x a l$ & $\mathrm{H}$ & $\mathrm{S}$ & 125,126 & Veracruz, Mpio. Chiconquiaco & $\begin{array}{l}\text { C. Gutiérrez } 3238 \text { (MEXU), M. Cházaro } \\
3574 \text { (MEXU) }\end{array}$ \\
\hline sar & $\mathrm{H}$ & $\mathrm{S}$ & 127,128 & Veracruz, Mpio. Coatepec & $\begin{array}{l}\text { J. I. Calzada } 2008 \text { (XAL), E. Estrada et. } \\
\text { col. } 770 \text { (ENCB) }\end{array}$ \\
\hline sar & $\mathrm{H}$ & $\mathrm{S}$ & 129 & Veracruz, Mpio. Cosautlán & R. Arriaga 199 (XAL) \\
\hline sar & $\mathrm{H}$ & $\mathrm{S}$ & 130 & Veracruz, Mpio. Coscomatepec & A. Lot 1182 (MEXU) \\
\hline
\end{tabular}


Apéndice 1. Continúa.

\begin{tabular}{|c|c|c|c|c|c|}
\hline Taxon & Material & Análisis & $\begin{array}{l}\text { Núm.de } \\
\text { referencia }\end{array}$ & Localidad & Colector \\
\hline sar & $\mathrm{H}$ & $\mathrm{S}$ & $131-138$ & Veracruz, Mpio. Huatusco & $\begin{array}{l}\text { M. Martínez } 2834 \text { (MEXU), S. Avendaño et } \\
\text { al. } 796 \text { (ENCB), S. Avendaño y J. I. Calzada } \\
522 \text { (XAL), G. K. Arp } 4184 \text { (XAL), E. } \\
\text { Guizar } 1511 \text { (ENCB), J. Rzedowski } 18938 \\
\text { (ENCB), D. C. López (CHAP), J. Rzedowski } \\
18931 \text { (ENCB) }\end{array}$ \\
\hline$x a l$ & $\mathrm{H}$ & $\mathrm{S}$ & 139,162 & Veracruz, Mpio. Huayacocotla & $\begin{array}{l}\text { J. Palma } 159 \text { (XAL), Y. A. Vargas } 368 \\
\text { (ENCB) }\end{array}$ \\
\hline sar & $\mathrm{H}$ & $\mathrm{S}$ & 140,141 & Veracruz, Mpio. Ixhuacán de Los Reyes & $\begin{array}{l}\text { M. Nee } 22475 \text { (ENCB), R. Arriaga y } H . \\
\text { López } 232 \text { (MEXU) }\end{array}$ \\
\hline sar & $\mathrm{H}$ & $\mathrm{S}$ & 142 & Veracruz, Mpio. Las Minas & C. Durán E. 1082 ( XAL) \\
\hline sar & $\mathrm{H}$ & $\mathrm{S}$ & 143 & Veracruz, Mpio. Naolinco & F. Ventura 8919 (ENCB) \\
\hline sar & $\mathrm{H}$ & $\mathrm{S}$ & 144 & Veracruz, Mpio. Tlalnehuayocan & M. G. Zola 652 (XAL) \\
\hline sar & $\mathrm{H}$ & $\mathrm{S}$ & 145 & Veracruz, Mpio. Totutla & C. Durán y T. Platas 1182 (XAL) \\
\hline sar & $\mathrm{H}$ & $\mathrm{S}$ & $146-155$ & Veracruz, Mpio. Xalapa & $\begin{array}{l}\text { M. G. Zola } 808 \text { (XAL), J. I. Calzada } 1927 \\
\text { (MEXU), M. G. Zola 600, } 809 \text { (MEXU), C. } \\
\text { G. Pringle } 8108 \text { (MEXU), A. P. Vovides } 60 \\
\text { (ENCB), M. Nee } 33068 \text { (XAL), L. Monroy } \\
69 \text { (XAL), F. Ventura } 2361 \text { (ENCB), A. P. } \\
\text { Vovides } 62 \text { (ENCB) }\end{array}$ \\
\hline sar & $\mathrm{H}$ & $\mathrm{S}$ & 156,161 & Veracruz, Mpio. Xico & $\begin{array}{l}\text { M. Cházaro y L. Robles } 2802 \text { (MEXU), F. } \\
\text { Ventura } 11623 \text { (ENCB) }\end{array}$ \\
\hline sar & $\mathrm{H}$ & $\mathrm{S}$ & $157-160$ & Veracruz, Mpio. Xilotepec & $\begin{array}{l}\text { J. Dorantes y M. Acosta } 1762 \text { (ENCB), } \\
\text { F. Ventura } 7117 \text { (ENCB), M. G. Zola } 435 \\
\text { (XAL), F. Ventura } 16976 \text { (MEXU) }\end{array}$ \\
\hline$x a l$ & $\mathrm{H}$ & $\mathrm{S}$ & 163 & Veracruz, Mpio. Huatusco & J. Rzedowski 19053 (ENCB) \\
\hline$x a l$ & $\mathrm{H}$ & $\mathrm{S}$ & 164 & Veracruz, Mpio. Soteapan & Guevara 15 (MEXU) \\
\hline sar & $\mathrm{H}$ & & & Veracruz, Mpio. Totutla & F. M. Liebmann 3554 (Tipo, NY) \\
\hline$x a l$ & $\mathrm{H}$ & & & Veracruz, Mpio. Xalapa & Bonpland 4467 (Tipo, MO) \\
\hline
\end{tabular}


Ap[endice 2. Caracteres y estados de carácter utilizados en los análisis multivariados y fenéticos de Quercus sartorii y Q. xalapensis. Los 10 caracteres utilizados en el DISCRIM se indican con un asterisco.

\begin{tabular}{|c|c|c|c|}
\hline Carácter & $\begin{array}{l}\text { Estado } \\
\text { de carácter }\end{array}$ & Carácter & Estado de carácter \\
\hline 1. Largo de la lámina foliar $(\mathrm{cm})^{*}$ & Media & 23. Número de células de la felodermis & Moda \\
\hline 2. Ancho de la lámina foliar $(\mathrm{cm})$ & Media & 24. Hoja lanceolada & Ausente (0); presente (1) \\
\hline 3. Largo del pecíolo $(\mathrm{cm})^{*}$ & Media & 25. Hoja elíptica & Ausente (0); presente (1) \\
\hline 4. Diámetro del pecíolo $(\mathrm{cm})$ & Media & 26. Hoja ovada & Ausente (0); presente (1) \\
\hline $\begin{array}{l}\text { 5. Longitud de las aristas de la parte } \\
\text { derecha de la lámina foliar }(\mathrm{cm})^{*}\end{array}$ & Media & 27. Hoja obovada & Ausente (0); presente (1) \\
\hline $\begin{array}{l}\text { 6. Longitud de las aristas de la parte } \\
\text { izquierda de la lámina foliar }(\mathrm{cm})^{*}\end{array}$ & Media & 28. Base de la hoja aguda & Ausente (0); presente (1) \\
\hline $\begin{array}{l}\text { 7. Ángulo medio de la parte derecha } \\
\text { de la lámina foliar }\left(^{\circ}\right)\end{array}$ & Media & 29. Base de la hoja atenuada & Ausente (0); presente (1) \\
\hline $\begin{array}{l}\text { 8. Ángulo medio de la parte } \\
\text { izquierda de la lámina foliar }\left(^{\circ}\right)\end{array}$ & Media & 30. Base de la hoja obtusa & Ausente (0); presente (1) \\
\hline 9. Longitud de las yemas $(\mathrm{cm})^{*}$ & Media & 31. Ápice agudo & Ausente (0); presente (1) \\
\hline 10. Ancho de las yemas $(\mathrm{cm})$ & Media & 32. Ápice acuminado & Ausente (0); presente (1) \\
\hline 11. Longitud de la bellota $(\mathrm{cm})$ & Media & 33. Borde de la hoja entero & Ausente (0); presente (1) \\
\hline 12. Diámetro de la bellota $(\mathrm{cm})^{*}$ & Media & 34. Borde de la hoja dentado & Ausente (0); presente (1) \\
\hline 13. Longitud de la cúpula $(\mathrm{cm})$ & Media & 35. Borde de la hoja aserrado & Ausente (0); presente (1) \\
\hline 14. Diámetro de la cúpula $(\mathrm{cm})^{*}$ & Media & 36. Bellota de forma globosa & Ausente (0); presente (1) \\
\hline 15. Longitud de la nuez $(\mathrm{cm})$ & Media & 37. Bellota de forma ovoide & Ausente (0); presente (1) \\
\hline 16. Diámetro de la nuez $(\mathrm{cm})^{*}$ & Media & 38. Base de la cúpula constreñida & Ausente (0); presente (1) \\
\hline $\begin{array}{l}\text { 17. Diámetro de la cicatriz de la nuez } \\
(\mathrm{cm})^{*}\end{array}$ & Media & 39. Base de la cúpula no constreñida & Ausente (0); presente (1) \\
\hline 18. Longitud del pedúnculo $(\mathrm{cm})$ & Media & 40. Arreglo de los vasos & $\begin{array}{l}\text { En hileras radiales }(0) \text {; en } \\
\text { diagonal (1); intermedio }\end{array}$ \\
\hline $\begin{array}{l}\text { 19. Proporción largo/diámetro de la } \\
\text { bellota }(\mathrm{cm})\end{array}$ & Media & 41. Aréolas de forma regular & (2) Ausente (0); presente (1) \\
\hline 20. Número de aréolas $\left(\mathrm{cm}^{2}\right)^{*}$ & Moda & 42. Vénulas simples & Ausente (0); presente (1) \\
\hline 21. Número de estomas $\left(\mathrm{cm}^{2}\right)$ & Moda & 43. Vénulas ramificadas 1 o 2 veces & Moda \\
\hline $\begin{array}{l}\text { 22. Largo de las células oclusivas } \\
(\mathrm{mm})\end{array}$ & Media & & \\
\hline
\end{tabular}

